\title{
¿QUÉ ES GERENCIA SOCIAL?: DEFINICIÓN DE LA CATEGORÍA' DESDE EL ANÁLISIS TEÓRICO.CRÍTICO
}

Freddy Esquivel Corella²

\author{
"Así, no es posible corregir una cuestión eminentemente política \\ con una corrección técnica, no siendo suficiente modernizar \\ el aparato profesional para resolver una cuestión \\ que no es meramente profesional “ \\ M. Iamamoto (1992: 62)
}

\section{RESUMEN}

El presente artículo retoma información bibliográfica y cualitativa sobre la aprehensión que se tiene desde diferentes fuentes, sobre la categoría gerencia social. Este artículo desarrolla un análisis por categorías de esta discusión, finalizando con una propuesta conceptual crítica

Palabras clave: Gerencia social - Trabajo Social.

\begin{abstract}
This article presents the análisis about the management social definitions. It had been done since qualitative information of bibliography sources, all this with the purpose to offer for readers a discusión over this issue at the end, it concluyes a critic approach concerning with social management.
\end{abstract}

Key words: Social management - Social Work

\footnotetext{
1 "Las Categorías son producto de la sociedad que las engendra, en un momento dado de su desarrollo" (Quiroga,1990:64).

${ }^{2}$ Licenciado en Trabajo Social (UCR). Magister Scientae en Trabajo Social con Énfasis en Investigación (SEP-UCR). Realiza estudios Doctorales en Educación (SEP-UNED) Docente Escuela y Maestría de Trabajo Social (UCR).
} 


\section{INTRODUCCIÓN}

D esarrollar el tema de la gerencia social desde una posición teórico-crítica es difícil y arriesgado a la vez. Difícil por el intento de colocar en la mesa de análisis una propuesta que ha tenido tanta acogida en el colectivo profesional, y que ha modificado sustancialmente la currícula universitaria, como la percepción de la profesión (su razón) en el marco de la sociedad actual.

Arriesgado, porque las interpretaciones que se hagan de este artículo, pueden estimar un mal manejo de sus conclusiones y aportes, al punto de que quienes lideran en las relaciones de poder que se engendran en el colectivo, no reconozcan el fortalecimiento de posiciones críticas sobre el tema en estudio, y continúen reproduciendo en la formación y en el ejercicio profesional, sin reflexión alguna, los aspectos que aquí se detallan.

El recurrir al tema de la gerencia social en una posición teórico-crítica, es una variante sobre los estudios que han predominado respecto al tema, por lo que el presente esfuerzo se articula a una serie de propuestas de estudio que han sido desarrolladas y discutidas por otros/as profesionales, y que se vinculan a la práctica de la profesión del Trabajo Social, desde una posición lejana a la conservadora dominante.

Este artículo incluye el análisis de las diversas definiciones que se han identificado en literatura consultada, como brindadas por expertos/as y profesionales entrevistados/as sobre el tema de la gerencia social.

La respuesta a la pregunta que abre este artículo facilitará primeramente ir delineando el camino que lleva un análisis de este tipo ${ }^{3}$, para luego profundizar en el estudio de esta categoría.

Paso entonces a exponer las diferentes definiciones identificadas:

\section{¿Qué es Gerencia Social? ${ }^{4}$}

\begin{tabular}{|c|c|c|}
\hline $\begin{array}{l}\text { R E F E R E N C I A S } \\
\text { BIBLIOGRÁFICAS SOBRE } \\
\text { GERENCIA SOCIAL }\end{array}$ & $\begin{array}{l}\text { Morera, 1995: Un proceso, donde } \\
\text { se busca el logro de los objetivos } \\
\text { de un servicio social, (enmarcado } \\
\text { en una institución pública o } \\
\text { privada), mediante determinada } \\
\text { tecnología (conocimientos, } \\
\text { técnicas, instrumentos, procedi- } \\
\text { mientos, materiales y equipos). }\end{array}$ & $\begin{array}{l}\text { Kliksberg (1995): La describe } \\
\text { como una profesión }{ }^{5} \text {, que propone } \\
\text { una crítica al modelo gerencial } \\
\text { tradicional, fundamentado en el } \\
\text { burocrátismo, para el desarrollo } \\
\text { de la política social, relacionándola } \\
\text { con la construcción de sociedades } \\
\text { democráticas activas, descentra- } \\
\text { lización del Estado, ampliación de } \\
\text { espacios de participación ciuda- }\end{array}$ \\
\hline
\end{tabular}

\footnotetext{
${ }^{3}$ Un análisis histórico crítico, es aquel que busca comprender la ontología, totalidad y génesis que se determinan en el objeto de análisis. Se intenta aprehenderlo en su relación con el modelo de producción capitalista especialmente, y su desarrollo histórico de manera dialéctica.

${ }^{4}$ Los aportes de personas que no aparecen entre comillas y cursivas, son apuntes tomados de sus declaraciones, ya que prefirieron no ser grabados, por lo que no hay transcripción de la entrevista.

${ }^{5}$ Pero aún en 1989, el autor señala esta propuesta como "administración social”, léase: "Las agudas consecuencias sociales de la crisis deberán ser paliadas en parte a través de programas de amplios
} 


\begin{tabular}{|c|c|c|}
\hline & & $\begin{array}{l}\text { dana, y de protección de los } \\
\text { derechos del/la ciudadano/a. }\end{array}$ \\
\hline $\begin{array}{l}\text { Molina (1995): Disciplina } \\
\text { científica, producto de un } \\
\text { vínculo entre las Ciencias } \\
\text { Sociales y la Administración, } \\
\text { que tiene cómo objeto de } \\
\text { estudio el análisis de institu- } \\
\text { ciones y la organización de la } \\
\text { producción, la gestión y } \\
\text { evaluación de los servicios } \\
\text { sociales y el impacto de estos en } \\
\text { el sistema social. }\end{array}$ & $\begin{array}{l}\text { Granell et al (1997) : Dirección } \\
\text { y administración de actividades de } \\
\text { naturaleza social, ubicadas tanto } \\
\text { en el sector público como en el } \\
\text { privado. }\end{array}$ & $\begin{array}{l}\text { Molina y Morera (1999): Un } \\
\text { medio, para producir servicios } \\
\text { sociales de calidad. }\end{array}$ \\
\hline $\begin{array}{l}\text { ALAETS-CELATS (1998): } \\
\text { Una técnica utilizada como } \\
\text { modelo alternativo de inter- } \\
\text { vención en Trabajo Social, y } \\
\text { como gerencia diferenciada. }\end{array}$ & $\begin{array}{l}\text { Vega (1995): Una estrategia para } \\
\text { la racionalidad en el uso de los } \\
\text { recursos destinados a lo social. }\end{array}$ & $\begin{array}{l}\text { Muñoz (1995): La gerencia social } \\
\text { es un modelo administrativo que } \\
\text { permite gestionar las acciones } \\
\text { sociales dentro de un marco de } \\
\text { eficiencia, eficacia e impacto, que } \\
\text { aporta instrumentos gerenciales } \\
\text { que pueden ser aplicados a } \\
\text { cualquier institución, y que no es } \\
\text { un proyecto político, sino un } \\
\text { modelo gerencial que recupera } \\
\text { elementos y tendencias de la } \\
\text { administración moderna y la } \\
\text { gerencia de servicios. }\end{array}$ \\
\hline
\end{tabular}

Fuente: Elaboración propia según fuentes consultadas.

\section{¿Qué es Gerencia Social?}

\begin{tabular}{|l|l|l|l|}
\hline DEFINICIONES DE & Exp. N.1"(...) es un movimiento, una & Exp. N.2"(...) una forma de \\
EXPERTOS /AS SOBRE & respuesta a ciertos fenómenos como la & conducir, de gerenciar, de administrar \\
GERENCIA SOCIAL & pobreza y la ausencia de desarrollo & servicios de carácter social, que pueden \\
acorde a nuestra situación. La gerencia & ser instituciones, programas, \\
social desde el punto de vista del & departamentos, Ongs, (...) gerenciar \\
INDES, es un esfuerzo de vincular lo & es un proceso de toma de decisiones, \\
económico y social, es una visión & que tiene centrar la atención en \\
integradora de cómo responder a las & enunciar la misión de cualquier servicio \\
necesidades sociales y económicas de & de carácter social, (...) gerencia social \\
la realidad." & también, puede tener muchas \\
& & $\begin{array}{l}\text { connotaciones, pero cuando yo bablo de } \\
\text { gerencia social, es centrada en servicios } \\
\text { dirigidos a poblaciones en condiciones } \\
\text { de pobreza (...)" }\end{array}$ \\
\hline
\end{tabular}

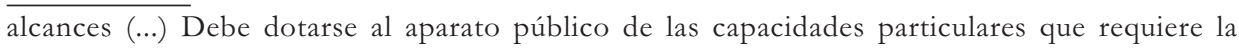
“administración social”, que son claramente diferenciadas de las pertinentes a otras formas de gestión” (Kliksberg, 1989: 27) (Negrilla no es del original). 


\begin{tabular}{|c|c|c|}
\hline $\begin{array}{l}\text { Exp. N.3 "(...) es un enfoque } \\
\text { alternativo para administrar, y para } \\
\text { conducir organizaciones productoras } \\
\text { de servicios sociales, y digo que es } \\
\text { alternativo, porque ésta es una } \\
\text { construcción muy tica y muy de } \\
\text { Trabajo Social, realmente lo que } \\
\text { ofrece es una visión distinta a los } \\
\text { enfoques de administración } \\
\text { tradicionales, con enfoques que } \\
\text { nosotros estábamos asumiendo, sin } \\
\text { más ni más, adaptados en el mejor } \\
\text { de los casos". }\end{array}$ & $\begin{array}{l}\text { Exp. N.4 "(...) administrar recursos } \\
\text { que están destinados, en un caso a } \\
\text { obtener rentabilidad, y en el otro a } \\
\text { generar programas y proyectos con } \\
\text { carácter solidario, equitativo, universal, } \\
\text { (...)". }\end{array}$ & $\begin{array}{l}\text { Exp. N.5 "(...) yo veo la gerencia } \\
\text { social, como un proceso, un conjunto de } \\
\text { procesos articulados, que esta dirigidos } \\
\text { a, organizar por una parte, todo lo que } \\
\text { tiene que ver en el caso de Trabajo } \\
\text { Social, con el diseño, la formulación, } \\
\text { la prestación y seguimiento de Servicio } \\
\text { Social (...)". }\end{array}$ \\
\hline $\begin{array}{l}\text { Exp. N.6 "(...) yo creo que bueno, } \\
\text { ha avanzado la disciplina de la } \\
\text { gerencia social en los últimos tiempos, } \\
\text { (...) primero, hablar de gerencia, es } \\
\text { hablar de acciones, o sea, no puede } \\
\text { uno concebir, si estamos hablando } \\
\text { del concepto, no puede uno concebir } \\
\text { una gerencia sin acciones, verdad, } \\
\text { en primer lugar, es un vinculo con } \\
\text { acciones, en segundo lugar, es un } \\
\text { vínculo con toma de decisiones, si } \\
\text { usted esos dos elementos los ve } \\
\text { analiticamente (...) se desprende de } \\
\text { esas ideas centrales de la acción y de } \\
\text { la toma de decisiones verdad,(...)" }\end{array}$ & $\begin{array}{l}\text { Exp. N.7 “(...) uno lo puede } \\
\text { conceptualizar como un campo } \\
\text { disciplinario y una práctica profesional, } \\
\text { que se ocupa fundamentalmente del } \\
\text { campo de la gestión de los servicios } \\
\text { sociales, sin fines de lucro, orientada } \\
\text { por valores fundamentales dirigidos al } \\
\text { bienestar de la población". }\end{array}$ & \\
\hline
\end{tabular}

Fuente: Elaboración Propia, según entrevistas semiestructuradas realizadas.

¿Qué es Gerencia Social?

\begin{tabular}{|l|l|l|}
\hline DEFINICIONES DE & T.S. N.1 “ (...) yo lo relaciono & T.S N.2 Gerencia social es la \\
TRABAJADORAS/ ES & $\begin{array}{l}\text { inmediatamente con población, con } \\
\text { SOCIALES SOBRE } \\
\text { GERENCIA SOCIAL } \\
\text { comunidades, con programas, con toda que puede tomar } \\
\text { esta intervención que es muy propia del } \\
\text { Trabajo Social.” } \\
\text { responsabilidad, que puede tomar } \\
\text { decisiones, que consulta as otras } \\
\text { instancias para poder aplicarlas, } \\
\text { tiene que tomar responsabilidades } \\
\text { que se puedan hacer, no cosas que } \\
\text { no se puedan hacer, la gerencia } \\
\text { social, es una idea que anda } \\
\text { volando en la academia pero que } \\
\text { en la realidad no se puede hacer. }\end{array}$ \\
\hline $\begin{array}{l}\text { T.S N.3"(..) la gerencia social (...) } \\
\text { es como el arte de poder utilizar una } \\
\text { serie de berramientas de tipo } \\
\text { metodológico, y también como }\end{array}$ & \\
\hline
\end{tabular}




\begin{tabular}{|c|c|c|}
\hline $\begin{array}{l}\text { manejar variables de poder como la } \\
\text { autoridad (...) yo diría también que } \\
\text { gerencia social, es también gestionar } \\
\text { o también conducir procesos que } \\
\text { lleven a soluciones innovativas, } \\
\text { creativas, adecuadas a las realidades } \\
\text { y a los contextos a los que deviene } \\
\text { esa acción (....)". }\end{array}$ & $\begin{array}{l}\text { T.S. N.4 Yo creo que, quizás es } \\
\text { una cuestión de habilidad, mucho } \\
\text { te lo da la experiencia, capacidad } \\
\text { de negociación y de llegar a } \\
\text { concretar. }\end{array}$ & $\begin{array}{l}\text { T.S N.5" (...) es la administración } \\
\text { que se hace de programas que tienen } \\
\text { que ver con una respuesta a un problema } \\
\text { social, que el Estado esta tratando de } \\
\text { dar,(...) todo lo que esta inserto dentro } \\
\text { del manejo de un programa social". }\end{array}$ \\
\hline $\begin{array}{l}\text { T.S. N.6 "Se trata de administrar } \\
\text { para alcanzar objetivos en un } \\
\text { programa social de interés social, } \\
\text { eso seria, digamos algo muy básico, } \\
\text { pero no desde paradigmas de gerencia } \\
\text { como se entiende en los negocios, en } \\
\text { la administración de negocios, sino } \\
\text { que desde ya con otros paradigmas, } \\
\text { que tienen que ver con que las } \\
\text { personas que son usuarias de los } \\
\text { servicios puedan tener, una atención } \\
\text { eficaz, eficiente, que sus derechos se } \\
\text { cumplan, que los servicios no sean } \\
\text { concebidos básicamente, como } \\
\text { regalias, si no que éste realmente } \\
\text { pueda tener servicios como un derecho } \\
\text { de distribución de beneficios en la } \\
\text { sociedad,(...)". }\end{array}$ & $\begin{array}{l}\text { T.S. N.7 “(...) es el manejo de } \\
\text { programas de carácter social, de } \\
\text { lineamientos sociales, no sé si estoy } \\
\text { equivocada, en realidad yo pienso que } \\
\text { estuve manejando o estuve } \\
\text { desarrollando gerencia social (...)". }\end{array}$ & \\
\hline
\end{tabular}

Fuente: Elaboración propia según entrevistas semiestructuradas realizadas.

De esta manera se destaca que las respuestas son muy diversas, y es en realidad compleja su definición; para ello se desarrolla a continuación una síntesis de los insumos antes expuestos, para caer luego en el análisis de éstas y la definición que propone este artículo.

\section{Síntesis de los insumos}

En resumen, la gerencia social ha sido definida según los resultados de esta investigación como:

- Una profesión o disciplina

- Traslado del "management" privado al sector público

- Un planteamiento apolítico

- Algo diferente a la administración

- Administración de servicios sociales

- Modelo, técnica o proceso.

- Medio

- Cargo 
- "Idea Académica".

- Cambio en la racionalidad de los servicios sociales.

Paso entonces a desagregar cada una de las anteriores categorías elaborando un análisis crítico sobre sus argumentaciones.

\section{Gerencia Social como Profesión y/o Disciplina}

Dentro de las definiciones de esta categoría, son varias las que perfilan la gerencia social como una profesión, y que la reconocen como una disciplina que se desarrolla en el marco de la crítica al Estado de bienestar a la administración pública burocrática y a la democratización, teniendo incluso una especificidad propia.

Similar a Kliksberg, la gerencia social es planteada en la definición de Molina (1995) con una independencia científica, y con un objeto propio de estudio, lo cual la demarcaría como una profesión y/o disciplina.

Lo anterior permite reconocer el poder que tienen las estructuras ideológicas de construir parcelaciones de la realidad social, a través de la constitución de categorías que reducen la complejidad de los conflictos sociales; ello, por medio de "nuevas profesiones", que de igual forma no rompen en lo absoluto con propuestas reduccionistas, ni con una racionalidad fragmentadora de la realidad social, y por ende fortaleciendo el no atravesar las mediaciones que se constituyen en ella.

Ante ello es necesario incorporar la necesidad de que la categoría Trabajo Social ${ }^{6}$, desde esta óptica de entender la gerencia social como disciplina o profesión, genere un debate que reconozca que esta nueva profesión viene legitimada por los organismos internacionales que la conciben y, por lo tanto, es producto del capitalismo, tal y como lo fue el Trabajo Social en la edad monopolista de dicho modo de producción.

Gerencia Social como Traslado del "Management" Privado al Sector Público

Se destaca igualmente en la bibliografía consultada, que hay una tendencia a considerar que gerencia social no es el traslado del "management" privado al público.

En realidad esta concepción del traslado de técnicas de "lo privado" a "lo público" no es muy fuerte en la gerencia social; de hecho son pocas las personas consultadas que hacen referencia a esta comprensión, que de paso es un reduccionismo instrumental de gran envergadura.

\footnotetext{
${ }^{6}$ De igual manera cabe la reflexión, de que si Trabajo Social se está vinculando a la gerencia social, lo está haciendo: ¿Para nutrirse de una nueva profesión? ¿Para, reconocer que los programas y proyectos sociales han sido impactados por el burocratismo al que se vinculó el desarrollo del capital? ¿Para generar propuestas de intervención diferenciadas, pero que no rompen con la racionalidad que se engendra en esta propuesta de gerencia social?; y una última pregunta: ¿No conocía gran parte del colectivo profesional las deficiencias en las políticas sociales, que hoy demarca la gerencia social?
} 
Esta propuesta también se ha mezclado con la idea de que este enlace de "lo privado" con "lo público”, en materia de administración, permitiría dar un perfil empresarial a las organizaciones productoras de servicios sociales, estimándose hasta rentabilidad (Ver Muñoz, 1996).

Lo que significa erróneamente que los servicios sociales estatales están aislados de la dinámica del capital, y que no tienen ninguna función de rentabilidad para las clases en el poder, Iamamoto (1992), señala sobre ello:

"Se torna un medio no sólo para mantener a la fuerza de trabajo en condiciones de ser explotada productivamente, evitando alteraciones substanciales en la política salarial que afectan la lucratividad de los empresarios” (pág. 108)

Cabe señalar que es de Kliksberg (1995), de quien muchos autores destacan la necesidad de una vinculación de la política social y la económica, pero valga anotar que ambas políticas, siempre han estado articuladas, ya sea para fomentar el consumo, como en el keynesianismo, o para orientar su inversión y ganancias a ciertos sectores de la sociedad, como sucede ahora con la privatización de servicios.

Lo que se hace aquí es una inversión de la realidad, intentando proponer la gerencia social como "rescatadora", en lugar de situarla en su razón de "reproductora" de las relaciones sociales imperantes ${ }^{7}$.

Es importante resaltar que el desarrollo de la gerencia social no sólo se establece en el sector público; su propuesta de implementación se orienta a las organizaciones no gubernamentales y comunales. En relación con las responsabilidades estatales, la gerencia social alega más bien una delegación de la búsqueda de la solución de sus problemas a las mismas personas; proyecta que las acciones de asistencia social se enmarcan más bien en una neofilantropía, y en relaciones de acceso al propio mercado, como satisfactor de esas demandas.

Tampoco es válido el pensar que la gerencia social, como propuesta, se dirige únicamente a comunidades, ya que como se ha demostrado, su planteamiento original es la inserción en programas y/o proyectos sociales, indiferentemente de las poblaciones con las que la organización o el servicio se vincule.

\section{Gerencia Social como Administración de Servicios Sociales}

En algunas definiciones se reconoce que la gerencia social es la administración de los recursos sociales hacia la satisfacción de las demandas de las poblaciones, comprendidas como derechos, y la búsqueda de la rentabilidad y socialización de los costos en los servicios sociales.

A pesar de la iniquidad de distribución de ganancias y la explotación de los/as trabajadores/ as, esta rentabilidad más que ir orientada a la búsqueda de la universalidad, la solidaridad y la

${ }^{7}$ En el Trabajo Social, se puede tender a reproducir esa "separación de lo económico con lo social", ya que en forma inmediata los/as profesionales en su contexto organizacional, pueden aducir que su trabajo no tiene ninguna incidencia en la dinámica económica, es únicamente con el estudio de las mediaciones que se da ese salto analítico de manera más enriquecida.

¿Qué es GeRenCia Social?: DEFinición de la Categoría ... / FREDdy ESQuivel CoRella 
equidad, se orienta en su propuesta original, a la focalización, o sea exclusión, negación y privatización de servicios sociales, que desde una óptica neoliberal lleva a lograr por un lado ganancias a los sectores privados que intervienen en la producción de servicios sociales. Por otro lado, conduce a que las personas asuman la resolución de sus demandas por medio del mercado, pero en una inexistencia de recursos económicos por parte de la mayoría de la población para satisfacerlas.

Con respecto a los costos en las políticas sociales, según el neoliberalismo, cabe señalar que aquí la asistencia social es concebida como un acto moral, y no como una responsabilidad del Estado hacia las poblaciones explotadas.

\section{Gerencia Social como planteamiento Apolítico}

Se destacó en la comprensión de la gerencia social, que ésta es un planteamiento apolítico, pero para efectos de este artículo se rechaza en forma definitiva esa apreciación, ya que la gerencia social, desde su proyecto original, está vinculada a la globalización neoliberal como proyecto político, o sea tiene toda una intencionalidad.

Una de las expertas entrevistadas señaló, sobre esta concepción apolítica de la gerencia social:

“(...) gerencia social, cómo un conjunto de procesos donde se establecen relaciones de poder,(...), pero cuando hablamos de esa toma de decisiones, suena como que eso esta en una cápsula vacía, que yo siendo objetiva, usando toda una racionalidad generalmente instrumental, entonces voy a poder tomar las decisiones más eficaces, hasta el momento resulta que la toma de decisiones esta mediada por una serie de elementos, verdad, que tienen que ver con el contexto, pero que tienen que ver también con relaciones de poder. A pesar de eso, que es lo que ha pasado con muchos enfoques como de los de gerencia social, que han terminado favoreciendo una reproducción justamente, de esas relaciones de poder, verdad, asimétricas, que producen exclusión, que producen discriminación, y a legitimar esa, discriminación, marginación, inclusive violencia. Estamos frente a la administración, por ejemplo, de la discriminación institucionalizada, la exclusión institucionalizada y la violencia institucionalizada” (Exp, N 5).

\section{Gerencia Social como algo diferente a la Administración}

Existe una tendencia a estimar la necesidad de diferenciar gerencia de administración, donde la primera, se supone que incluye un manejo más político, de interacción con la incertidumbre, de acceso a información, que la administración no desarrolla, como también el potenciar la descentralización, buscar la "participación", preocuparse mayormente por resultados, que la hagan diferenciarse de una "administración burocrática".

La administración por su parte se ubica en un esquema menos flexible, más determinado y programado.

El punto no es quizás hacer esta demarcación tan determinante de una y otra; se considera que si bien el gerenciar requiere una serie de destrezas, habilidades y conocimientos; estos van a 
estar igualmente bañados, como la administración de racionalidad instrumental e inmediatez, ya que si el profesional no logra trascender a la reconstrucción de su práctica e intervención, se continúa con la reproducción de intervenciones instrumentalistas e inmediatas.

\section{Gerencia Social como Modelo, Técnica o Proceso}

También se plantea en la revisión bibliográfica una confusión, donde la gerencia social es modelo y es técnica; a ello cabe apuntar que al reducirla a técnica, se le resta a dicha propuesta toda una serie de elementos constitutivos ${ }^{8}$, especialmente de la propuesta original de Kliksberg, que desdibujan su funcionalidad político-ideológica, y si se acepta como modelo vendría a caer una vez más en la idea de poder tener "recetas" de intervención profesional.

Vale agregar al respecto un aporte de una de las entrevistadas donde dice que:

“(...) yo puedo plantearme, que siento que es mucho el problema, la gente o vienen con un modelo ya armado que lo tiene legitimado, eso no es sólo con gerencia social, es con terapia, cuando reducen a técnica la gerencia social, (...), verdad, entonces yo tengo la cosa armadita, ese es el paso uno, paso dos, elpaso tres, verdad" ( Exp. N.5).

Se pone en relieve aquí que la gerencia social también es comprendida como un conjunto de procesos articulados, dirigidos en el caso del Trabajo Social, al diseño, la formulación, y la prestación y seguimiento del servicio social; en dichos procesos se establecen relaciones de poder; cita también la entrevistada que se concibe a la gerencia social muchas veces con una racionalidad instrumental que busca tomar decisiones eficaces.

Entenderla como proceso no pone como fundamento la tarea estratégica que se desarrolla con la gerencia social que si bien está permeada por relaciones de poder, que es un elemento poco rescatado en la literatura consultada, no es vinculada en esta óptica con su labor instrumental; pero basada en la racionalidad lógico formal-abstracta, o sea, ese proceso tiene toda una racionalidad de fundamento, que desde el origen mismo de la gerencia social no va a romper con ella, y por ende no va a facilitar reconocer las mediaciones que se engendran en ese proceso y su vinculación al desarrollo capitalista.

\section{Gerencia Social como Medio}

Igualmente, la gerencia social fue reconocida en las entrevistas como un "medio", lo cual pone en cuestión toda la finalidad a la cual está sirviendo como propuesta articuladora del desarrollo neoliberal.

Desde la óptica de este artículo, dicha creación de los organismos internacionales, es más que un medio, y no es un fin, o sea más que un recurso; se proyecta como un legitimador de un

\footnotetext{
${ }^{8}$ A pesar de ello se debe comprender que toda técnica también tiene un sustento ideológico político y teórico-metodológico.
}

¿Qué es Gerencia Social?: definición de la Categoría ... / FREdDy esQuivel corella 
proyecto que intenta delegar muchas de las responsabilidades del Estado en las poblaciones.

$\mathrm{Al}$ reconocérsele como un medio, debe entenderse que hay que trascender la instrumentalidad con la cual se encuentra investida, para definirla y comprenderla en el momento histórico de manera más integral.

\section{Gerencia Social como Cargo}

En una entrevista se estimó que la gerencia social se logra definir por sus atribuciones, dadas por un cargo determinado de "Gerente/a Social"; lo anterior es limitante porque extrae su intervención tanto de un marco organizacional, generalmente jerárquico y patriarcal, donde se engendran una serie de mediaciones que determinan en forma significativa su labor, sea en una organización estatal, privada, pública o comunal.

\section{Gerencia Social como "Idea Académica"}

El reclamo de los profesionales entrevistados sobre concebirla como una "idea académica” se hizo sentir en el trabajo de campo, lo que pone de relieve las fuertes limitaciones que tiene su implementación en algunas organizaciones.

Se desea destacar en esta misma línea, que dentro de algún sector de la población de Trabajo Social, se han introducido una serie de elementos que "intentan diferenciar" la propuesta de gerencia social originada de los organismos internacionales, pero definirla únicamente desde dicha profesión, deja por fuera la naturaleza original de ésta, que es un planteamiento más difundido y desarrollado en la realidad concreta del continente.

Por lo que en este artículo el interés es categorizarla desde su origen en el seno del Banco Interamericano de Desarrollo - Instituto de Desarrollo Social, y con el aporte de Bernardo Kliksberg, y por ende a la búsqueda de crear condiciones para el desarrollo del capitalismo transnacionalizado.

Las propuestas que se orienten a estudiar las definiciones que se gestan únicamente en el Trabajo Social, asumen una postura endogenista, muy característica para comprender elementos constitutivos de la profesión. Pero para tener una visión más amplia de la gerencia social, debe profundizarse en la propuesta original de Kliksberg.

\section{Gerencia Social como Cambio de Racionalidad en los Servicios Sociales}

Finalmente es importante resaltar que el hecho de que se considere que en la gerencia social se varía la racionalidad, que es una propiedad de la razón, es un error, ya que ella se mantiene sobre sus mismos fundamentos lógico-formales, lo que varía es la distribución y direccionalidad de la asistencia social, respondiendo ahora a las demandas neoliberales.

Si bien es cierto que el manejo de los recursos es modificado, ahora más focalizado, excluyente y limitado, no se dirige a buscar una mejoría en la reproducción de los/as trabajadores/as, por el contrario los/as empobrece y somete más a las lógicas de explotación. 


\section{Una definición Teórico-Crítica de la Gerencia Social}

Luego de haber hecho ese recorrido sobre las definiciones de esta categoría, se desarrolla la definición de gerencia social que guiaría un análisis teórico-crítico de esta categoría.

Inicialmente debe destacarse que la gerencia social es una propuesta estratégica que se ha constituido en una "área de formación”, con el desarrollo de toda una estructura educativa y de asesoría, para conformar personal preparado que enfrente los recortes hacia la inversión en el "sector social".

Dentro de las tareas de la gerencia social, se encuentra el mantener la reproducción de las políticas redistributivas imperantes en el capitalismo, y generar estructuras y prácticas que faciliten ir desarrollando, por un lado, la maximización del mercado en la vida social, y por otro, el proceso de reforma del Estado (ya no de bienestar), que requiere el capitalismo en la onda recesiva que se encuentra.

Bajo esta coyuntura se gesta un fortalecimiento a la instrumentalidad amparada en la lógica formal imperante en la propuesta capitalista, y una reproducción de la inmediatez de sus cuadros profesionales.

Igualmente la gerencia social realiza una inversión de lo que se desarrolla en su vinculación con el capital, en lugar de propiciar una "unión entre lo social y lo económico", fortalece las relaciones de predominancia de crear condiciones de reproducción del modo, antes que el bienestar de las poblaciones.

En el escenario de la globalización neoliberal y de las transformaciones de la producción de los servicios sociales, la gerencia social toma partido para integrar bajo un discurso consecuente con la exigencia de avances democráticos en América Latina, la delegación de las responsabilidades estatales en materia de asistencia social, a las poblaciones y a las personas.

Para efecto de este trabajo, se reconoce además que los servicios sociales se encuentran inmersos en la dinámica del capital, desde sus políticas redistributivas, hasta la extracción de la plusvalía a la población trabajadora, pero el peso de las contradicciones constitutivas de las políticas sociales, por la relación capital-trabajo, se agravan en el actual escenario.

Esta propuesta de gerencia social también tiene un fundamento claro en la crítica al burocratismo que el mismo capitalismo desarrolló en un momento de su historia, y que ahora requiere ir modificando, pero no eliminando, así que la crítica a la burocracia de los servicios sociales, demanda "modernizar" estructuras y procedimientos, pero jamás racionalidades.

Según lo anterior la categoría gerencia social es definida en este artículo como:

'Una propuesta del desarrollo del neoliberalismo especialmente en las economias periféricas para enfrentar la escasez de recursos destinados hacia la redistribución social, por medio de las políticas sociales capitalistas, que se encubren en acciones que alegan intentar disminuir las asimetrías sociales generadas a partir de diferencias "naturales" entre las personas y sus relaciones en la sociedad y el mercado. 
Articulándose al desarrollo de la globalización neoliberal (y a las transformaciones en el modelo de producción y la reforma del Estado), la gerencia social - que descansa en la racionalidad lógico formal para el abordaje de la "cuestión social”, y que tiene un alto componente de traspaso de la lógica del Estado a la lógica de la sociedad civil- se enlaza a una participación social despolitizada y deseconomizada (reproductora de la inmediatez); que intenta hacer crítica al modelo burocrático tradicional, sin lograr trascender los fundamentos de éste".

\section{CONCLUSIONES}

En este artículo se comprobó que no existía un análisis teórico-crítico de las definiciones de la categoría gerencia social.

Esta reconstrucción permitió tener mayores insumos para el análisis presentado y para abonar a la discusión sobre la gerencia social en el Trabajo Social.

Según la información de las entrevistas semiestructuradas, la revisión documental y la investigación bibliográfica, la gerencia social ha sido definida de múltiples maneras, sea como profesión, disciplina científica, modelo, técnica, proceso, medio, estrategia, respuesta, enfoque, puesto, arte, habilidad.

Pero en ninguna de las definiciones se hace alusión directa a su vínculo con la racionalidad en que se sostiene que es la lógico formal para el abordaje de la "cuestión social”, enlazándose a una participación social despolitizada y deseconomizada; por ende reproductora de la inmediatez.

Queda entonces la tarea de trascender la discusión sobre lo que el gremio de Trabajo Social esta validando como Gerencia Social.

\section{REFERENCIAS BIBLIOGRÁFICAS}

ALAETS-CELATS (1998) "Conclusiones XV Seminario Latinoamericano de Trabajo Social”. Revista Acción Crítica N. 36-37.ALAETS-CELATS.Lima Perú.

Gallardo, Helio (1996) “Neoliberalismo en América Latina”. Ponencia presentada en el I Congreso

Universitario Internacional de Trabajo Social. San José, Costa Rica.

GRANELl DE ALDAZ, ELENA ET AL (1997) Elperfil del gerente social. Fundación Escuela de Gerencia Social.

Venezuela.

IAMAMOTO, MARILDA (1992) Servicio Social y División del Trabajo. Editorial Cortez. São Paulo, Brasil. KLiksBerg, Bernardo (1989) ¿Cómo transformar al Estado? Fondo de Cultura Económica. México. KLIKsberg, Bernardo (1995) Pobreza el drama cotidiano: clave para una nueva Gerencia Social eficiente.

CLAD-Grupo Edtorial Norma. PNUD. Buenos Aires, Argentina.

LAROUSE (Diccionario) (1985). Barcelona, España.

MOLINA, LORENA (1995) “Trabajo Social y Gerencia de Servicios Sociales”. Ponencia presentada en el

XV Seminario Latinoamericano de Trabajo Social. "Nuevos Escenarios y Desafíos para el Trabajo

Social". Guatemala.

MOLINA, LORENA y MORERA, NIDIA (1999) La Gerencia de los Servicios Sociales. Editorial Lumen/Hvamnitas.

Buenos Aires, Argentina.

MORERA, NIDIA (1995) “La Gerencia Social: Herramienta Indispensable para la Conducción de Servicios

Sociales en el Umbral del Siglo XXI" en Revista Costarricense de Trabajo Social N.5. Colegio de

Trabajadores Sociales de Costa Rica. San José, Costa Rica. 
MUÑoz, MARÍA (1995) Gerencia Social. Centro de Apoyo al Sector Informal. Lima Perú.

MUÑOZ, MARÍA (1996) "Gerencia Social, una propuesta para el desarrollo" en Gerencia Social. Una Alternativa para el desarrollo humano. Cuadernos de Trabajo Social N.3.CONAETS, Colombia.

QUIROGA, CONSUELO (1990) "Una invasión invisible: Reducciones positivistas en el marxismo y sus manifestaciones en la enseñanza de metodología en Servicio Social", en Revista Acción Crítica N.28. ALAETS-CELATS. Lima, Perú.

VEGA, CECILIA (1995) "Una perspectiva histórica del Estado, las políticas sociales y el Trabajo Social en Costa Rica”, en Revista de Trabajo Social. N ${ }^{\circ}$ 44, año 21 Caja Costarricense de Seguro Social, San José, Costa Rica.

Recibido en mayo 10 de 2004

Aprobado en junio 15 de 2004 